PHYSICAL REVIEW D 93, 039902(E) (2016)

\title{
Erratum: Probing lepton nonuniversality in tau neutrino scattering [Phys. Rev. D 92, 073016 (2015)]
}

Hongkai Liu, Ahmed Rashed, and Alakabha Datta

(Received 21 January 2016; published 5 February 2016)

DOI: $10.1103 /$ PhysRevD.93.039902

This erratum serves to give corrections for two errors made in Liu, Rashed, and Datta.

First error: The constraints on the new physics parameters of the $V \pm A$ model are taken from the single and double pion decay modes of the tau lepton, $\tau^{-} \rightarrow \nu_{\tau}+\pi^{-}$and $\tau^{-} \rightarrow \nu_{\tau}+\pi^{-}+\pi^{0}$. The constraint plots included in the paper show the effect of the single pion decay only, while it is mentioned in the captions that both modes are considered. Incorrect plots were uploaded with correct captions. The correct figures which include the effects of both decay modes are shown below in Figs. 1 and 2. Contrary to the original results, the constraints on the $V \pm A$ model parameters are more stringent when considering both decay modes.

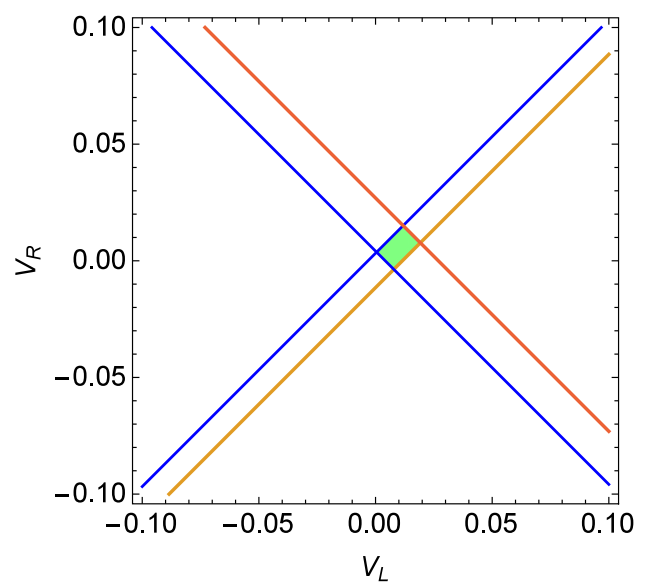

FIG. 1. The allowed region (green area) for the left- and right-handed couplings $V_{L}$ and $V_{R}$. The constraints are from $\tau^{-} \rightarrow \pi^{-} \nu_{\tau}$ and $\tau^{-} \rightarrow \pi^{-} \pi^{0} \nu_{\tau}$.
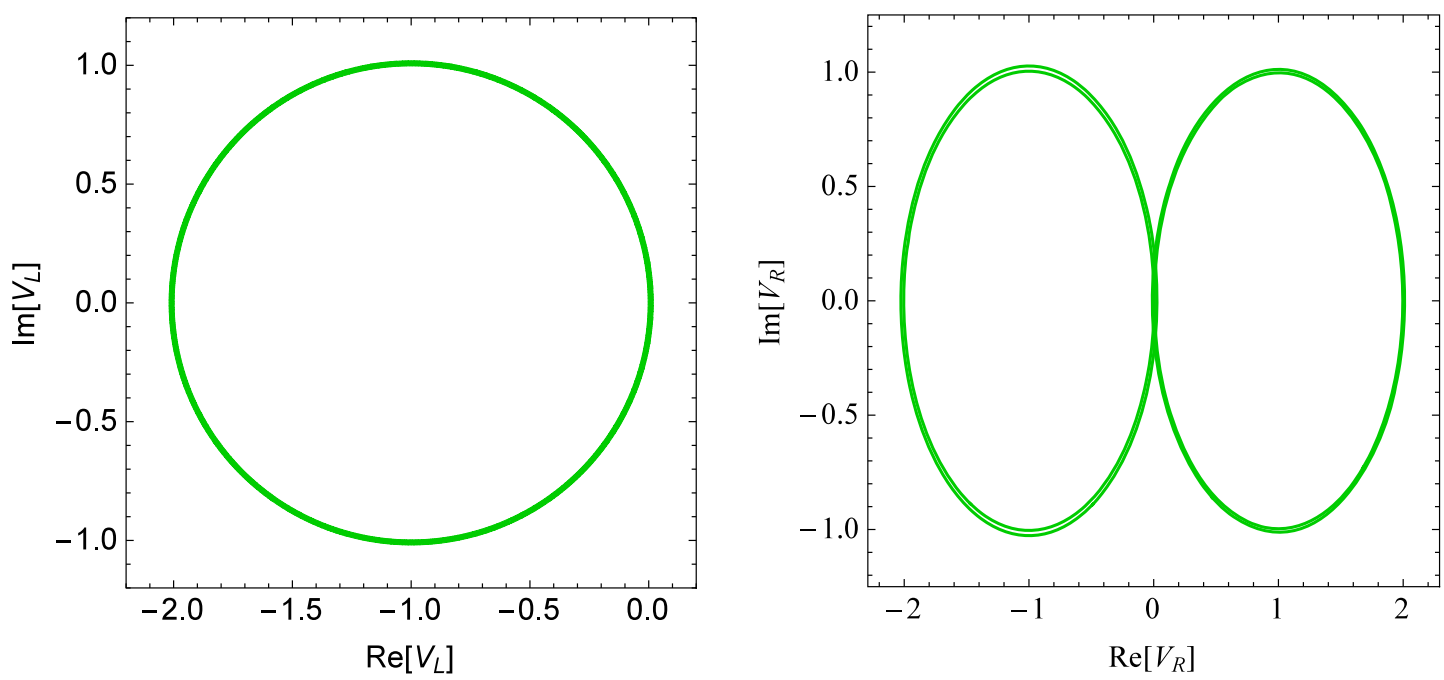

FIG. 2. The allowed regions for the left- and right-handed complex couplings $V_{L}$ and $V_{R}$. The constraint are from $\tau^{-} \rightarrow \pi^{-} \nu_{\tau}$ and $\tau^{-} \rightarrow \pi^{-} \pi^{0} \nu_{\tau}$. Left panel: We take $V_{R}=0$ and treat $V_{L}$ as a complex coupling. The allowed region is the contour ring. Right panel: We take $V_{L}=0$ and treat $V_{R}$ as a complex coupling. The allowed region is the overlap area between the two contour rings. 




FIG. 3. $V \pm A$ model: The ratio between the total cross section of $\nu_{\tau}+N \rightarrow \tau+X$ to $\nu_{\mu}+N \rightarrow \mu+X$ in the $V \pm A$ model. The green line corresponds to the standard model predictions $V_{L}=V_{R}=0$. The red dot-dashed line corresponds to $\left(V_{L}, V_{R}\right)=(0.016,0.006)$.

Second error: The new physics parameters used in producing the cross section and differential cross section distributions for the neutrino-nucleon scattering in the paper are constrained by tau decay to single pion only. Incorrect plots were uploaded which show large nonstandard interaction (NSI) effects. The correct plots below (Figs. 3, 4, 5, 6, 7, and 8) show that the new physics effects in the scattering cross section are small because the

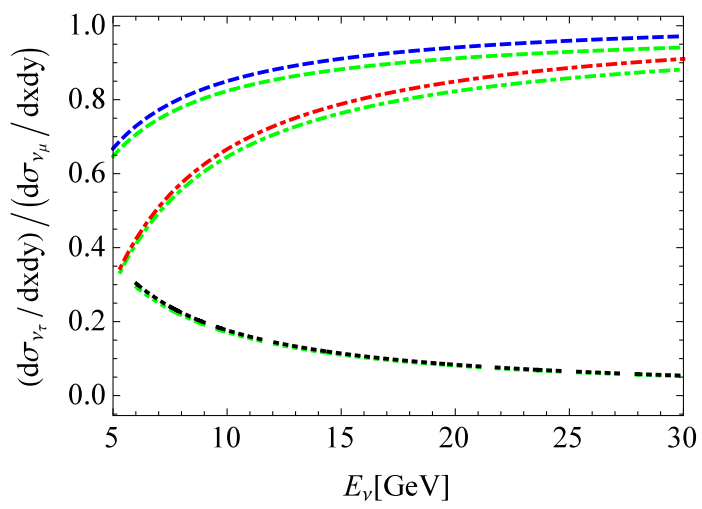

FIG. 4. $V \pm A$ model: The ratio between the differential cross section $(d \sigma / d x d y)$ of $\nu_{\tau}+N \rightarrow \tau+X$ to $\nu_{\mu}+N \rightarrow \mu+X$ in the $V \pm A$ model. The green lines correspond to the standard model predictions $V_{L}=V_{R}=0$. The blue, black, and red lines correspond to $\left(V_{L}, V_{R}\right)=(0.016,0.006)$. The blue and green dashed lines correspond to $(x, y)=(0.95, A+B)$. The red and green dot-dashed lines correspond to $(x, y)=(0.475,(A+B) / 2)$. The black and green dotted lines correspond to $(x, y)=\left(\frac{m_{\tau}^{2}}{2 M\left(E_{\nu}-m_{\tau}\right)}, A-B\right)$.

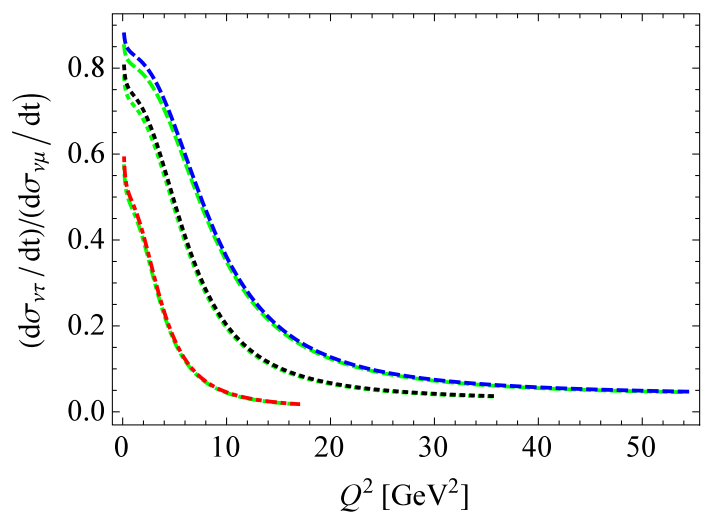

FIG. 5. $V \pm A$ model: The ratio between the differential cross section $(d \sigma / d t)$ of $\nu_{\tau}+N \rightarrow \tau+X$ to $\nu_{\mu}+N \rightarrow \mu+X$ in the $V \pm A$ model. The green lines correspond to the standard model predictions $V_{L}=V_{R}=0$. The blue, black, and red lines correspond to $\left(V_{L}, V_{R}\right)=(0.016,0.006)$ at $E_{\nu}=30,20$, and $10 \mathrm{GeV}$, respectively. The physical region of the momentum transfer is taken to be $Q_{-}^{2}\left(W_{\text {cut }}\right) \leq Q^{2} \leq Q_{+}^{2}\left(W_{\text {cut }}\right)$. 




FIG. 6. $V \pm A$ model: The total cross section of $\nu_{\tau}+N \rightarrow \tau+X$ in the $V \pm A$ model. The green line corresponds to the standard model predictions $V_{L}=V_{R}=0$. The red dot-dashed line corresponds to $\left(V_{L}, V_{R}\right)=(0.016,0.006)$.

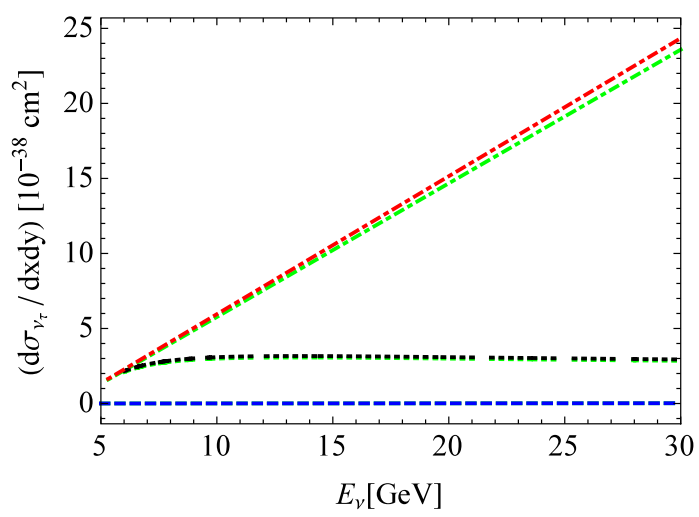

FIG. 7. $V \pm A$ model: The differential cross section $(d \sigma / d x d y)$ of $\nu_{\tau}+N \rightarrow \tau+X$ in the $V \pm A$ model. The green lines correspond to the standard model predictions $V_{L}=V_{R}=0$. The blue, black, and red lines correspond to $\left(V_{L}, V_{R}\right)=(0.016,0.006)$. The blue and green dashed lines correspond to $(x, y)=(0.95, A+B)$. The red and green dot-dashed lines correspond to $(x, y)=(0.475,(A+B) / 2)$. The black and green dotted lines correspond to $(x, y)=\left(\frac{m_{\tau}^{2}}{2 M\left(E_{\nu}-m_{\tau}\right)}, A-B\right)$.

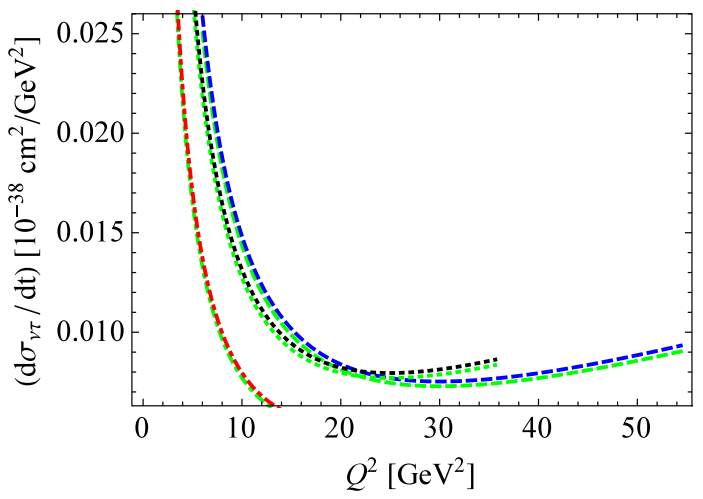

FIG. 8. $V \pm A$ model: The differential cross section $(d \sigma / d t)$ of $\nu_{\tau}+N \rightarrow \tau+X$ in the $V \pm A$ model. The green lines correspond to the standard model predictions $V_{L}=V_{R}=0$. The blue, black, and red lines correspond to $\left(V_{L}, V_{R}\right)=(0.016,0.006)$ at $E_{\nu}=30,20$, and $10 \mathrm{GeV}$, respectively. The physical region of the momentum transfer is taken to be $Q_{-}^{2}\left(W_{\text {cut }}\right) \leq Q^{2} \leq Q_{+}^{2}\left(W_{\text {cut }}\right)$. 
NSI parameters are strongly constrained when considering the two tau decay modes. The corrected forms of Sec. IV C and the plots can be found below.

\section{C. $V \pm A$ Interactions}

The ratio of the total cross section, $d \sigma / d x d y$, and $d \sigma / d t$ are shown in Figs. 3, 4, and 5, respectively. The figures show that the effects of $V \pm A$ new physics are small in the neutrino cross sections. The new physics effects are small in the total cross section, $d \sigma / d x d y$, and $d \sigma / d t$ for the process $\nu_{\tau}+N \rightarrow \tau+X$, as shown in Figs. 6, 7, and 8 . 